\title{
Chaos and emittance growth due to nonlinear interactions in a circular accelerator
}

\author{
K. Ohmi and K. Oide \\ KEK, 1-1 Oho, Tsukuba, 305-0801, Japan \\ (Received 23 October 2006; published 30 January 2007)
}

\begin{abstract}
Incoherent emittance growth caused by a strong nonlinear interaction between beam and electron cloud is discussed. This emittance growth arises from nonlinear diffusion related to chaos and resonances, and strongly depends on the number of degrees of freedom of the interacting system. A simple model, in which beam particles interact with a fixed round charge distribution, is used to study the mechanism of the emittance growth. The same discussion can be applied to the emittance growth due to beam-beam interactions in colliders and space charge effects in low energy proton rings.
\end{abstract}

DOI: 10.1103/PhysRevSTAB.10.014401

PACS numbers: 29.20.Dh, 29.27.- a, 41.75.Ht

\section{INTRODUCTION}

Emittance is one of the most fundamental quantities for a beam in a circular accelerator. It characterizes the size of the beam in the phase space of each degree of freedom, $x$, $y$, and $z$. It is important for the accelerator to maintain the design emittance during the operation. In high luminosity or high intensity machines, strong nonlinear interactions due to beam-electron cloud, beam-beam, and space charge forces cause emittance growth, with the result that accelerator performance is limited. Emittance growth is classified broadly into two categories, coherent or incoherent. Incoherent emittance growth due to nonlinear interactions is the subject of this paper.

Emittance is closely related to an invariant of motion for the particles in a beam. In a linear system, the motion of particles is described by a transformation using a $6 \times 6$ matrix of their coordinates $\left(x, p_{x}, y, p_{y}, z, p_{z}\right)$. Three invariants corresponding to the degrees of freedom, $x, y$, and $z$, so-called Courant-Snyder invariants $(2 \boldsymbol{J})$, exist for the linear transformation. The emittances $\left(\varepsilon_{x}, \varepsilon_{y}, \varepsilon_{z}\right)$ are defined as an average of one-half of the Courant-Snyder invariant all over the beam particles for $x, y$, and $z\left(\left\langle J_{i}\right\rangle\right)$.

Nonlinear interactions influence the nature of the invariants and/or emittances. We focus on the nonlinear interaction between a bunch and electron cloud, in which an enormous number of beam and cloud particles interact with each other. The so-called weak-strong model, in which beam particles interact with a fixed charge distribution, is used; i.e., the interaction can be expressed by a static potential and each particle moves in the potential independently. The fundamental physics of emittance growth in the beam-electron cloud interaction is the same as those in the beam-beam and space charge interactions. Work has been done in these fields in the 1970s-1980s [1$3]$ and is continued in the 2000s [4-6]. Recent work has been done relying on computer simulations.

We study interactions between a round beam and an electron cloud. Electrons are focused and pinched by the beam force with axial symmetry along the bunch passage. Beam particles experience a strong nonlinear force while preserving axial symmetry and a dependence on $z$ from the cloud. The axial symmetry makes clear what is important for the emittance growth. The number of degrees of freedom for the interacting system can be controlled by a choice of parameters as is shown later.

The Hamiltonian which characterizes the motion of beam particles is expressed by

$$
H\left(x, p_{x}, y, p_{y}, z, p_{z}, s\right)=H_{0}+U(r, z) \delta_{P}(s),
$$

where $H_{0}$ is the Hamiltonian for the linearized motion, i.e., betatron and synchrotron motion. $U$ is the potential for the interaction with the charge distribution with axial symmetry, and $\delta_{P}(s)$ the periodic delta function for revolution, $\sum_{n=1}^{\infty} \delta(s-n)$, where the circumference of the ring is normalized, $L=1$. Beam particles interact with the charge distribution at a location $(s=0+n)$ in this model. The number of degrees of freedom of the interacting system is reduced to $4(x, y, z, s)$, as single particle motion in a potential.

Emittance was defined by an average of "invariants" $(\boldsymbol{J})$ for $H_{0}$. The invariants $(\boldsymbol{J})$ are not true invariants for the nonlinear system including $U$. We have to consider the true invariants of the nonlinear system. The invariants correspond to integrals of the equation of motion. If we have three true invariants $(\bar{J})$, it means that the equation of motion is solved, and the true invariants are expressed as functions of the invariants and betatron phase of the linear system $(\overline{\boldsymbol{J}}(\boldsymbol{J}, \boldsymbol{\phi}))$. Particles in the beam are distributed as a function of $\overline{\boldsymbol{J}}, \Psi(\overline{\boldsymbol{J}})$. Emittance can be defined as an average of $\boldsymbol{J}$, though it deviates from that given in the linear system and is not an exact invariant,

$$
\varepsilon_{i}=\int J_{i} \Psi[\bar{J}(\boldsymbol{J}, \phi)] d \boldsymbol{J} d \boldsymbol{\phi} .
$$

We have to note that the invariants do not necessarily exist in a nonlinear system with two or more degrees of freedom. It means that there is no trajectory determined by a functional relation like $\overline{\boldsymbol{J}}(\boldsymbol{J}, \boldsymbol{\phi})=$ const. Particles may behaves stochastically, and emittance, the average of the "invariant" $(\boldsymbol{J})$, has the nature of diffusion; that is, emit- 
tance grows as a function of time like the solution of the diffusion equation

$$
\varepsilon_{y}(t) / \varepsilon_{y}(0) \approx 1+D t,
$$

where $D$ is the diffusion rate. Needless to say, the increase of the emittance, which results from complex nonlinear dynamics, does not necessarily behave clearly linearly over time.

Emittance growth, so-called "diffusion," is one of the important topics of classical chaos dynamics. Diffusion results from the superposition of the complex motion of independent single particles. Some particles behave chaotically but others do not. It is important to understand the single particle motion, while the diffusion rate, which is an averaged quantity over all particles, is a direct measure of the chaotic behavior for the purpose of our study.

The emittance growth and/or diffusion due to nonlinear interaction is studied using the weak-strong model. We show the number of degrees of freedom is important for the emittance growth. First, the interaction model and the simulation method using the weak-strong model are introduced in Sec. II. Emittance growth in systems with 2, 3, and 4 degrees of freedom, and in a more realistic model, are discussed in Secs. III, IV, V, and VI, respectively.

\section{MODEL OF THE INTERACTION}

The motion of the beam is determined by the linear oscillation term $\left(H_{0}\right)$ and the potential term $(U)$, which characterizes the interaction between beam and electron cloud, as shown in Eq. (1). The linear oscillation term is given by

$$
\begin{aligned}
H_{0}= & \frac{1}{2}\left(p_{x}^{2}+p_{y}^{2}\right)+\frac{1}{2}\left[K_{x}(s) x^{2}+K_{y}(s) y^{2}\right] \\
& -\frac{\alpha}{2}\left[p_{z}^{2}+\left(\frac{\mu_{z}}{\alpha}\right)^{2} z^{2}\right],
\end{aligned}
$$

where $K, \alpha$, and $\mu_{z}$ are the magnetic focusing force, the momentum compaction factor, and the synchrotron tune, respectively.

The linear map from exit to entrance to the interaction point is expressed as a $6 \times 6$ matrix,

$$
\begin{aligned}
M & =\left(\begin{array}{ccc}
M_{x} & 0 & 0 \\
0 & M_{y} & 0 \\
0 & 0 & M_{z}
\end{array}\right) \\
M_{i} & =\left(\begin{array}{cc}
\cos \mu_{i} & \beta_{i} \sin \mu_{i} \\
-\sin \mu_{i} / \beta_{i} & \cos \mu_{i}
\end{array}\right),
\end{aligned}
$$

where $\beta_{i}$ is the beta function of the interaction point and $\beta_{z}=\sigma_{z} / \sigma_{p_{z}} \cdot \mu_{i}$, which is the phase advance of the transformation, is related to the beta (synchrotron) tune by $\mu_{i}=$ $2 \pi \nu_{i} . \mu_{z}\left(\nu_{z}\right)$ is negative for positive momentum compaction, $\alpha>0$.
Focusing on the one-turn map, the effective Hamiltonian is expressed as

$$
H_{0}=\mu_{x} J_{x}+\mu_{y} J_{y}+\mu_{z} J_{z}
$$

$J_{x}$, which is the action variable, $2 \pi J_{x}=\oint p_{x} d x$, is expressed as

$$
J_{x}=\frac{1}{2}\left(\beta_{x} p_{x}^{2}+2 \alpha_{x} p_{x} x+\gamma_{x} x^{2}\right),
$$

where the same expressions for $J_{y}$ and $J_{z}$ with Twiss parameter, $\beta_{i}, \alpha_{i}$, and $\gamma_{i}$ are given.

The potential is assumed to be induced by a round Gaussian charge distribution with rms size $\Sigma_{r}(z)$, that is, it is expressed as

$$
U\left[r ; \Sigma_{r}(z)\right]=-\frac{N_{e} r_{e}}{\gamma} \int_{0}^{\infty} \frac{\exp \left(-\frac{r^{2}}{2 \Sigma_{r}(z)^{2}+u}\right)}{2 \Sigma_{r}(z)^{2}+u} d u .
$$

The size of the electron cloud changes in $z$ by the pinching due to the beam force. It is changed by the hourglass effect for beam-beam interaction. For the space charge effect, though the size is constant, the charge line density is a function of $z$, and $N$ should be replaced with $N \rho(z)$, where $\rho(z)$ is the normalized charge line density along the bunch length. Anyway, there is no difference in respect to the fact that $U$ is a function of $z$.

The variables $(r, \theta)$ are sometimes more convenient to use than $x, y$, for interactions with round symmetry. The canonical momenta for $r, \theta$ are expressed as

$$
\begin{aligned}
& p_{r}=r^{\prime}=p_{x} \cos \theta+p_{y} \sin \theta \\
& p_{\theta}=r^{2} \theta^{\prime}=r\left(-p_{x} \sin \theta+p_{y} \cos \theta\right) .
\end{aligned}
$$

The transformation of the momenta at the interaction point $(s=0+n)$ is expressed by

$$
\begin{aligned}
\Delta p_{r}=-\frac{\partial U}{\partial r} & =-\frac{2 N r_{e}}{\gamma} \frac{1}{r}\left[1-\exp \left(-\frac{r^{2}}{2 \Sigma_{r}^{2}}\right)\right], \\
\Delta p_{z} & =-\frac{\partial U}{\partial z}=-\frac{\partial U}{\partial \Sigma_{r}^{2}} \frac{d \Sigma_{r}^{2}}{d z} \\
& =-\frac{2 r_{e}}{\gamma} \frac{1}{2 \Sigma_{r}^{2}} \exp \left(-\frac{r^{2}}{2 \Sigma_{r}^{2}}\right) \frac{d \Sigma_{r}^{2}}{d z}
\end{aligned}
$$

The transformations for $p_{x}$ and $p_{y}$ are obtained by the relation of Eq. (9).

An accelerator model based on the LHC parameter is used for numerical analysis: $\varepsilon_{x}=\varepsilon_{y}=8 \times 10^{-9} \mathrm{~m}, \varepsilon_{z}=$ $6.1 \times 10^{-5}, \beta_{x}=\beta_{y}=100 \mathrm{~m}, \beta_{z}=278 \mathrm{~m}, \sigma_{x}=\sigma_{y}=$ $\sigma_{r}=0.89 \mathrm{~mm}$, and $\sigma_{z}=0.13 \mathrm{~m}$.

The charge distribution interacting with the beam is assumed to be Gaussian with rms size as a function of $z$,

$$
\rho(r, z)=N_{e} / 2 \pi \exp \left[-r^{2} / \Sigma_{r}(z)^{2}\right],
$$

where 


$$
\Sigma_{r}(z)=\sigma_{r} \sqrt{\left(0.05+z^{2} / 0.05\right)} .
$$

The size is the same as the beam size at $z= \pm 1.7 \sigma_{z}$, and has a minimum value $0.22 \sigma_{r}$ at $z=0$ in this model. $N_{e}$ is the number of electrons which interact with the beam in the ring with a circumference $(L=26700 \mathrm{~m})$. The corresponding tune shift is plotted in Fig. 1 together with the size. The peak tune shift is $\xi \equiv \Delta \nu_{\beta \text {, peak }}=0.06375$, $0.1275,0.255,0.3825$ for $N_{e}=1,2,4,6 \times 10^{11}$, respectively. The tune shift parameter $(\xi)$ is used to characterize the strength of the potential hereafter. The tune shift value corresponds to the cloud density $\rho_{e}=5 \times 10^{11} \mathrm{~m}^{-3}$ in the area, $5 \sigma_{r}, N_{e}=\pi\left(5 \sigma_{r}\right)^{2} \rho_{e} L=3.3 \times 10^{11}$. We also note that the size of the charged distribution is $\sim 1 / 5$ of the beam size at the peak tune shift.

The emittance growth can be found as an averaged quantity of independent single particle motions. Both studies of single particle motion and multiparticle tracking are performed. A Poincare plot is used to study the single particle motion. Several particles, ten or less, are initialized for some set of conditions, for example $r=0.2 j \times \sigma_{r}$, where $j=1$ to 10 , while keeping $p_{\theta}$. The particles are tracked using the transformations of Eqs. (5), (10), and (11). Particle coordinates are plotted in terms of $r-p_{r}$ at the interaction point. This plot, which is called a Poincaré surface of section or Poincaré plot, gives information on the chaotic nature of the particle.

Another approach is the calculation of the diffusion rate defined by Eq. (3) using multiparticle tracking. Many particles generated randomly are tracked and the average size of their distribution is calculated turn by turn. The diffusion rate is estimated by the gradient of the size increase.

The emittance growth for a system with 2 to 4 degrees of freedom is discussed in the following four sections. In the rest of this section, we briefly discuss the system with 1 degree of freedom. For the system with 1 degree of freedom, emittance growth is caused by the potential well

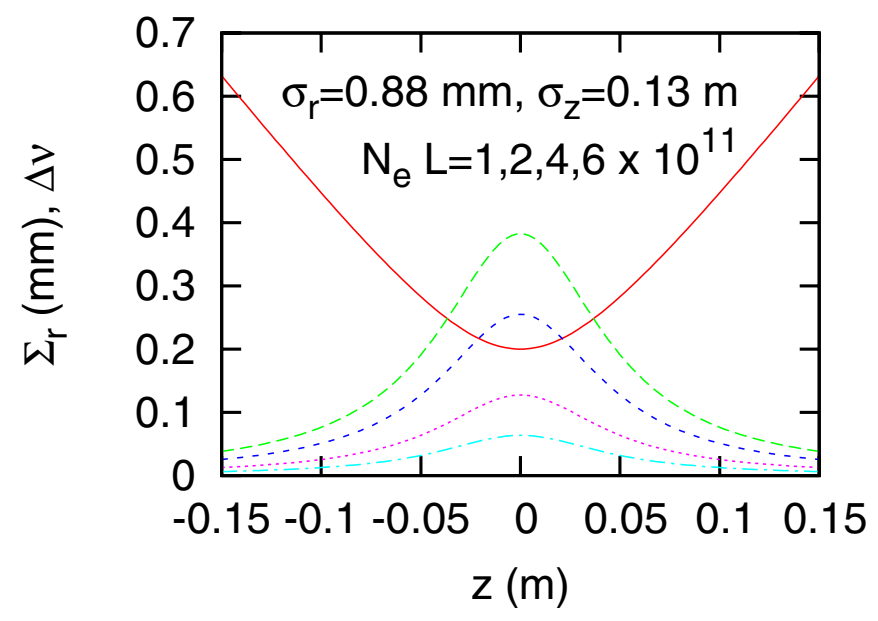

FIG. 1. (Color) Model of cloud size and tune shift. distortion. Since synchrotron motion is slow in most accelerators, the longitudinal motion is determined by the integrated potential $(U(z))$ along the ring: i.e., independent of $s$. As is well known, a system with 1 degree of freedom is integrable. The Hamiltonian is expressed by the summation of those of the synchrotron motion and the potential, $H=H_{0}+U(z)$. The invariant is $H$ and the action variable $\left(J_{z}\right)$ is represented by a function of $H . \bar{J}_{z}$ is distorted due to the potential $(U)$ from $J_{z}$ determined by $H_{0}$. An initial beam distribution is given by $\Psi_{0}\left(J_{z}\right)$, while the true static distribution including nonlinearity must be $\bar{\Psi}\left(\bar{J}_{z}\right)$. An emittance growth or change results from the difference of the two distributions, $\Psi_{0}\left(J_{z}\right)$ and $\bar{\Psi}\left(\bar{J}_{z}\right)$.

\section{SYSTEM WITH TWO DEGREES OF FREEDOM}

A system with 4 degrees of freedom, $x, y, z, s$, which is described by Eq. (1), can be reduced to one with 2 degrees of freedom by choosing parameters. That is, the horizontal tune and beta function are chosen to be equal to the vertical ones, and the synchrotron tune is zero. The integer part of the tune and beta function away from the interaction point do not need to be worried about; that is, it is not necessary that $K_{x}(s)=K_{y}(s)$ in Eq. (4). The effective Hamiltonian is expressed by

$$
H_{0}=\mu\left[\frac{\beta}{2}\left(p_{r}^{2}+\frac{p_{\theta}^{2}}{r^{2}}\right)+\frac{r^{2}}{2 \beta}\right] .
$$

We know the solution for $H_{0}$, i.e., a particle moves along an ellipse with the center $x=y=0$ in the $x-y$ plane.

The action variable is defined for a noninteracting system $(U=0)$. The Hamiltonian in Eq. (14) does not contain $\theta$, therefore the angular momentum $p_{\theta}$ is a constant of motion. The action variable $J_{\theta}$ is equal to $p_{\theta}$,

$$
J_{\theta}=\frac{1}{2 \pi} \oint p_{\theta} d \theta=p_{\theta}
$$

$J_{r}$ is obtained as

$$
\begin{aligned}
J_{r} & =\frac{1}{2 \pi} \oint p_{r} d r=\frac{1}{\pi} \int_{r_{\min }}^{r_{\max }} \sqrt{\frac{2 E_{0}}{\mu \beta}-\frac{r^{2}}{\beta^{2}}-\frac{\ell^{2}}{r^{2}}} d r \\
& =E_{0} / 2 \mu-\ell / 2,
\end{aligned}
$$

where $E_{0}$ and $\ell$, which are the values of $H_{0}$ of $J_{\theta}$, are the energy and angular momentum, respectively. The Hamiltonian is expressed by the action variables as follows:

$$
H_{0}=2 \mu J_{r}+\mu J_{\theta}
$$

Note that the phase advance for $r$ is $2 \mu$, because the particle trajectory is an ellipse in the $x-y$ plane with center at $r=0$.

The action angles corresponding to the actions are expressed by 


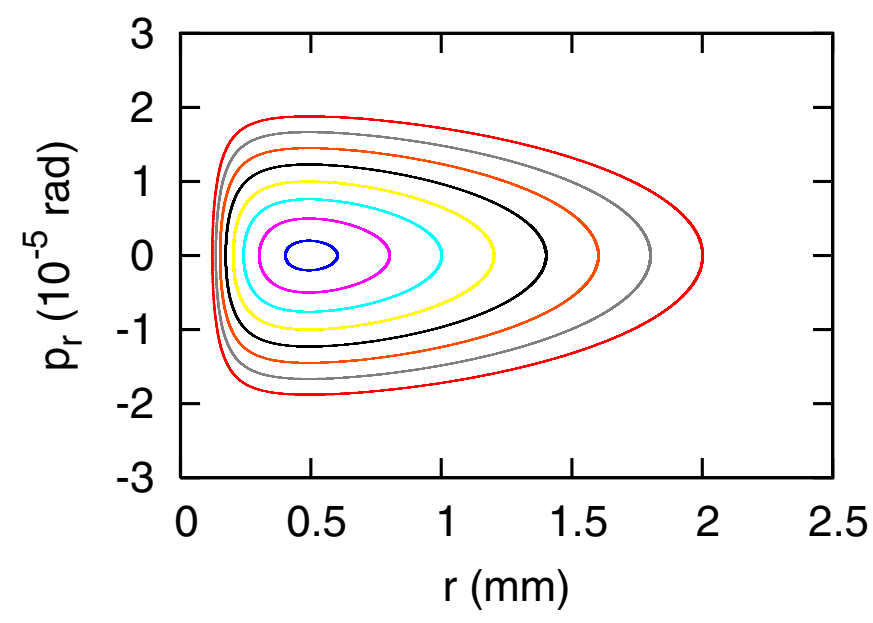

FIG. 2. (Color) Poincaré plot in $r-p_{r}$ for linear system, $U=0$.

$$
\psi_{\theta}=\theta \quad \psi_{r}=\cos ^{-1} \frac{r^{2}-\beta\left(2 J_{r}+\ell\right)}{\beta \sqrt{\left(2 J_{r}+\ell\right)^{2}-\ell^{2}}} .
$$

The addition of $U$ does not break the invariance of $p_{\theta}$. The system is reduced to one with 2 degrees of freedom, $r$ and $s$. If we have two integrals, the system is solved. One integral is trivial and formal, i.e., it is related to $H$ as a function of $s$. One variable $\left(p_{s}\right)$, which is the conjugate of $s$, is removed formally by the integral. The motion can be characterized by three variables, $r, p_{r}$, and $s$. The system is solved by the existence of another integral, $\bar{J}_{r}\left(r, p_{r}, s\right)=$ const. This relation for $\bar{J}_{r}$ gives a torus in $r-p_{r}-s$ space. Note that $H$ is periodic for $s+n \cdot \bar{J}_{r}=$ const gives a closed curve on $r-p_{r}$ phase space for a certain $s$. Conversely, a closed curve in the phase space, which is given in a numerical calculation, indicates the existence of the integral. The Poincaré plot gives information on the integrability of the system.

If the integral $\bar{J}_{r}\left(r, p_{r}, s_{0}\right)$ exists, the beam distribution $\Psi\left(\bar{J}_{r}\right)$ is maintained for the revolution $\left(s=s_{0}+n\right)$, with the result that the emittance $\left(\left\langle\bar{J}_{r}\right\rangle\right)$ is also maintained, except for distortion of $\bar{J}_{r}$ from $J_{r}$. It is essential for the emittance growth whether $\bar{J}_{r}$ exists or not.
We investigate the integrability of the model in Eqs. (1) and (14) via the Poincaré plots. We first show the Poincaré plot for $H_{0}$ in Fig. 2. The horizontal and vertical tunes are $\nu_{x}=\nu_{y}=0.2901$, and particles are tracked over 10000 turns. Ten particles are tracked with initial conditions, $x_{0}=0.2 \times j \mathrm{~mm}$, where $j=1$ to $10, p_{x, 0}=y_{0}=0$ and $p_{\theta}=x_{0} p_{y, 0}=2.4 \times 10^{-9} \mathrm{~m}$. Ten closed curves with different initial conditions are seen in the figure. The closed curves are also obtained by the invariant $J_{r}$ as follows:

$$
2 J_{r}+\ell=\frac{1}{\beta} r^{2}+\beta\left(p_{r}^{2}+\frac{\ell^{2}}{r^{2}}\right)=\frac{x_{0}^{2}}{\beta}+\beta \frac{\ell^{2}}{x_{0}^{2}} .
$$

The motion of the particles is distorted by the interaction term $(U)$. The particles are tracked for some peak tune shift parameters, $\xi=0.06,0.13,0.27,0.38$, with the same conditions as those of Fig. 1. Figure 3 shows the Poincaré plot with the same initial condition as in Fig. 2. For the lowest tune shift $(\xi=0.06)$, the trajectories in phase space in Fig. 3(a) are slightly distorted from that without interaction (Fig. 2), but seem to still be solid curves. Island structures appear at $\xi=0.13$, and some solid curves disappear for $\xi>0.13$ as shown in 3(b) $-3(d)$. The large five islands seen in the figure are induced by tenth order resonance [see Eq. (17)]. This feature means that the system is basically nonintegrable. Some solid trajectories are found according to the $\xi$ parameter and the initial amplitude. The nature of integrability does not depend on $\xi$ nor the initial amplitude. The solid curves mean that the system is approximately integrable at these parameters. This situation, in which a closed trajectory is found, is called "near integrable."

For some parameter values, solid trajectories disappear; , therefore the system is clearly nonintegrable. For higher tune shifts $(\xi \geq 0.27)$, the particle trajectories are scattered in a region, i.e., particles seem to move randomly in the region. This region is called the stochastic layer. The phase space consists of the stochastic layer and the near integrable region for high tune shifts. A higher tune shift gives a wider stochastic area.

For the "near integrable" case, an approximate invariant $\left[\bar{J}_{r}\left(r, p_{r}\right)\right]$ exists. If all beam particles are the near inte-

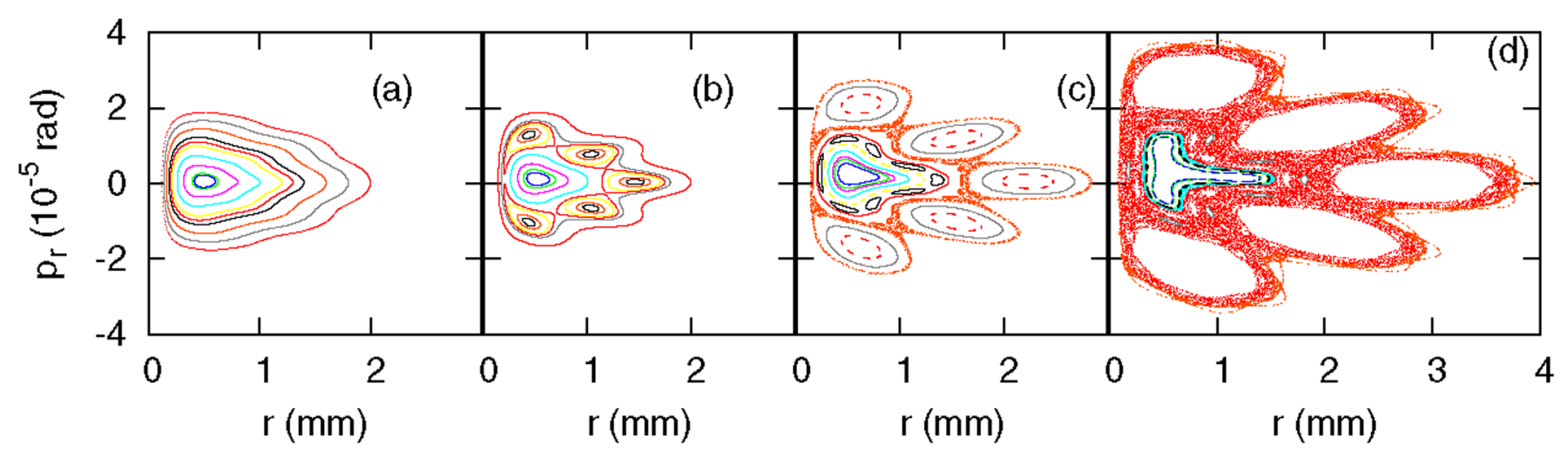

FIG. 3. (Color) Poincaré plot in $r-p_{r}$ space for various tune shift values: (a) $\xi=0.06$, (b) $\xi=0.13$, (c) $\xi=0.27$, and (d) $\xi=0.38$. 
grable region, a distribution $\Psi\left(J_{r}\right)$ determined by the initial condition is kept for the revolution, though $\bar{J}_{r}$ is distorted from $J_{r}$ due to $U$. Especially when a particle is near a resonance island, the distortion is very large, with the result that the emittance defined by $\left\langle J_{r}\right\rangle$ can grow.

In the stochastic layer, an initial distribution is smeared out and the particle distribution is roughly flat. This process can be a source of the emittance growth. It is important that particles cannot jump from a near integrable curve to another near integrable curve or stochastic layer; that is, the emittance growth is limited, unless a very wide stochastic layer is formed by a very large tune shift.

The appearance of the wide stochastic layer is understood as the overlap of resonances. The resonance term is extracted by the Fourier expansion of the $U$ as follows:

$$
U(r)=\sum_{k=0}^{\infty} U_{k}\left(J_{r}\right) \cos \psi_{r}
$$

The Fourier coefficient characterizes the strength of the resonance,

$U_{k}\left(J_{r}\right)=\frac{2 N r_{e}}{\gamma} \int_{0}^{a} \frac{d w}{w}\left\{\delta_{k 0}-e^{-w} I_{k}\left[\sqrt{1-\frac{\ell^{2}}{\left(2 J_{r}+\ell\right)^{2}}} w\right]\right\}$,

where $a=\beta\left(2 J_{r}+\ell\right) / 2 \sigma_{r}^{2}$.

The tune shift due to the interaction term is expressed as a function of $J_{r}$ as follows:

$$
\bar{\mu}\left(J_{r}\right)=\mu+\frac{\partial U_{0}}{\partial J_{r}} .
$$

The amplitude $\left(J_{r, R}\right)$, at which the $k$ th order resonance appears, is evaluated as

$$
\bar{\mu}\left(J_{r, R}\right)=2 \pi n / k,
$$

where $n$ is an arbitrary integer.

New variables are introduced to represent the motion around the resonance:

$$
I_{r}=\frac{J_{r}-J_{r, R}}{k} \quad \theta=k \psi_{r} .
$$

The Hamiltonian with these variables is expressed as

$$
H=\frac{1}{2} k^{2} \frac{\partial^{2} U_{0}}{\partial J_{r}^{2}} I_{r}^{2}+\delta_{P}(s) U_{k}\left(I_{r, R}\right) \cos \theta,
$$

where the derivative for $U_{0}$ is evaluated at $J_{r}=J_{r, R}$. This Hamiltonian is pendulumlike, except that the potential term depends on time $(s)$ through $\delta_{P}(s)$. Particles experience a harmonic oscillation near $\theta=0$ with a small $I_{r}$. For a large amplitude $\left(I_{r}\right)$, particles move along a separatrix with a long period. A stochastic region is formed near the separatrix. The width of the separatrix is given by

$$
\Delta J_{r}=k \Delta I_{r}=4 \sqrt{U_{k}\left(\frac{\partial^{2} U_{0}}{\partial J_{r}^{2}}\right)^{-1}} .
$$

When the width of the separatrix approaches the separation from another separatrix, a stochastic layer can develop strongly. One typical example of the separation is $J_{r, R}(k, n+1)$, and $J_{r, R}(k, n)$ is narrower than the width. The separation is

$$
J_{r, R}(k, n+1)-J_{r, R}(k, n)=\frac{2 \pi}{k}\left(\frac{\partial^{2} U_{0}}{\partial J_{r}^{2}}\right)^{-1} .
$$

The criterion, which is called the Chirikov criterion, is expressed as follows:

$$
k \sqrt{U_{k} \frac{\partial^{2} U_{0}}{\partial J_{r}^{2}}}>\frac{\pi}{2} .
$$

There are also other criteria, for example, for different $k$.

Emittance growth is investigated by tracking with many particles. Particles are initialized with a Gaussian distribution with rms values determined by the emittance and beta function,

$$
\Psi_{0}\left(J_{r}\right)=\frac{N_{p}}{2 \pi} \exp \left(-\frac{J_{r}}{\varepsilon_{r}}\right) .
$$

The second order moments, $\left\langle x^{2}\right\rangle$ and $\left\langle y^{2}\right\rangle$, are calculated turn by turn. Figure 4 shows the evolution of $\left\langle y^{2}\right\rangle$. The emittance growth is small even for $\xi=0.38$, and the growth is saturated at an increase of $10 \%$ even for the highest tune shift, $\xi=0.38$.

This type of emittance growth, which is seen in the system with 2 degrees of freedom, is too simplified and is too weak. Such a large tune shift is not achievable in actual accelerators. Particles in accelerators are in a system with more degrees of freedom, therefore emittance growth is greater and more severe as shown later.

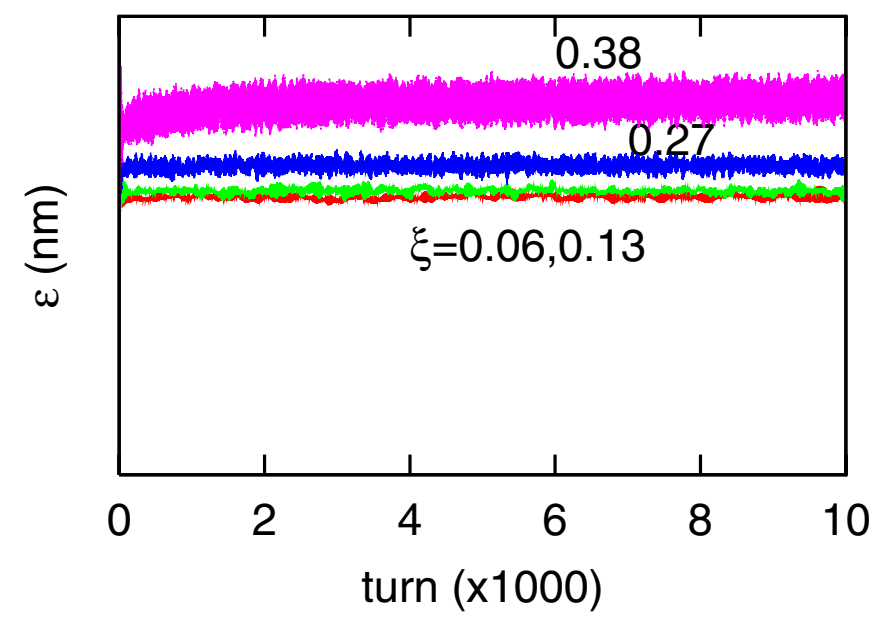

FIG. 4. (Color) Emittance growth for the model with 2 degrees of freedom for various tune shift parameters, $\xi=$ $0.06,0.13,0.27,0.38$. 


\section{SYSTEM WITH THREE DEGREES OF FREEDOM}

There are two ways to increase the number of the degrees of freedom. One is the introduction of synchrotron motion. It is essential that the interaction term $U(r, z)$ be a function of $z$, otherwise synchrotron motion does not contribute to an increase in the number of degrees of freedom. The other is the introduction of a difference between the horizontal and vertical tunes.

\section{A. Synchrotron motion}

The Hamiltonian including synchrotron motion is expressed as follows:

$H=2 \mu_{r} J_{r}+\mu_{r} J_{\theta}-\frac{\alpha}{2}\left[p_{z}^{2}+\left(\frac{\mu_{z}}{\alpha}\right)^{2} z^{2}\right]+\delta_{P}(s) U(r, z)$

The motion can be characterized by five variables, $\left(r, p_{r}, z, p_{z}, s\right)$. The system is integrable if we find two integrals, $\bar{J}_{i}\left(r, p_{r}, z, p_{z}, s\right)=\mathrm{const}$, where $i=1,2$. The particle trajectories can be projected on to $r-p_{r}$ space at a certain $s$. Even if the system is integrable, the trajectories are distributed in $r-p_{r}$ space, because the solution is expressed as $f\left(r, p_{r}, z\right)=$ const. Figure 5 shows $r-p_{r}$ plots with the same conditions as Fig. 3 for $\nu_{z}=0.006$. It is not easy to get useful information, integrable or not, from the plots.

The synchrotron motion is very slow compared to that of the betatron motion, and the longitudinal emittance $\left(\varepsilon_{z}\right)$ is much larger than the transverse ones $\left(\varepsilon_{x}\right.$ and $\left.\varepsilon_{y}\right)$. For the LHC, the values are $\nu_{s}=0.006$ and $\varepsilon_{x} / \varepsilon_{z}=1.3 \times 10^{-4}$. The synchrotron motion may be considered as an external motion, and an adiabatic invariance may be expected for the betatron motion. Figure 6 shows the phase space plot in the plane of $z=2,4,6,10 \mathrm{~mm}$ for $\xi=0.38$. If an adiabatic invariance is satisfied, particles pass across each $r-$ $p_{r}$ plane while keeping an invariant. The trajectories for $z=0,2,4$ are strongly chaotic, while that for $z=10 \mathrm{~mm}$ is a solid curve, and that for $z=6 \mathrm{~mm}$ is intermediate. Position and size of the resonance islands, 5th, 3rd, and others, change for $z$ and the stochastic layer becomes narrow for a larger $z$. These behaviors are due to the fact that the nonlinear force is weaker for a larger $z$ amplitude.

Trajectories of particles pass across the separatrices of the resonances or stochastic region during a synchrotron period. The adiabatic invariant disappears at the crossing of the stochastic region or an unstable fixed point of the separatrix, therefore the existence of the invariant cannot be expected for a high tune shift, $\xi \geq 0.13$.

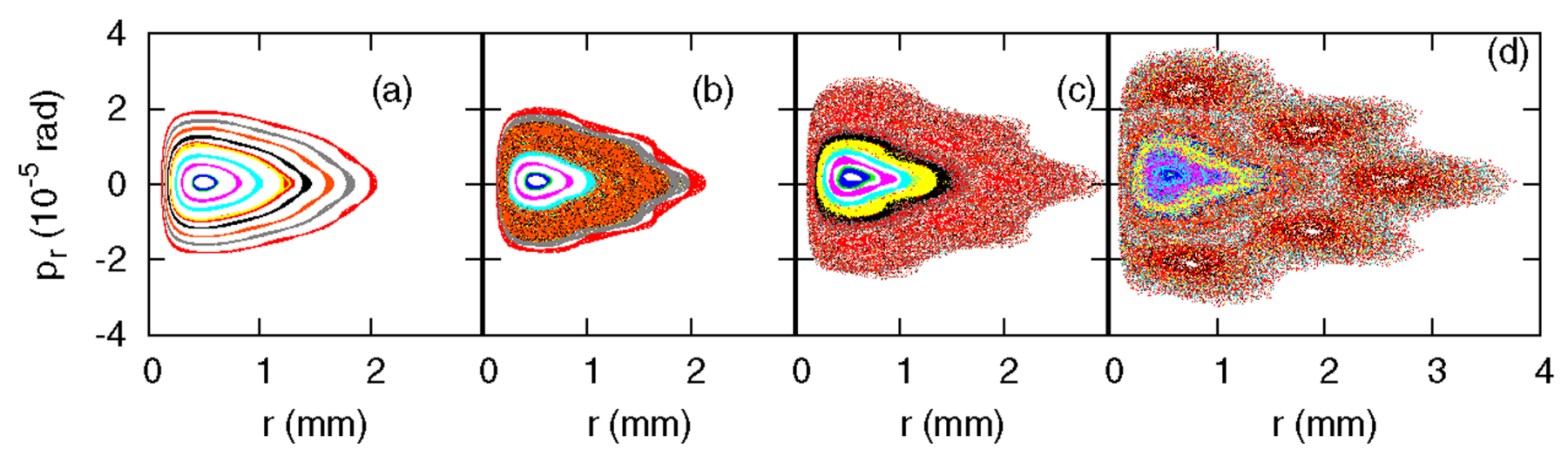

FIG. 5. (Color) Poincaré plot in $r-p_{r}$ space for various tune shift values: (a) $\xi=0.06$, (b) $\xi=0.13$, (c) $\xi=0.27$, and (d) $\xi=0.38$. Particles experience synchrotron oscillation with tune $\nu_{z}=0.006$.

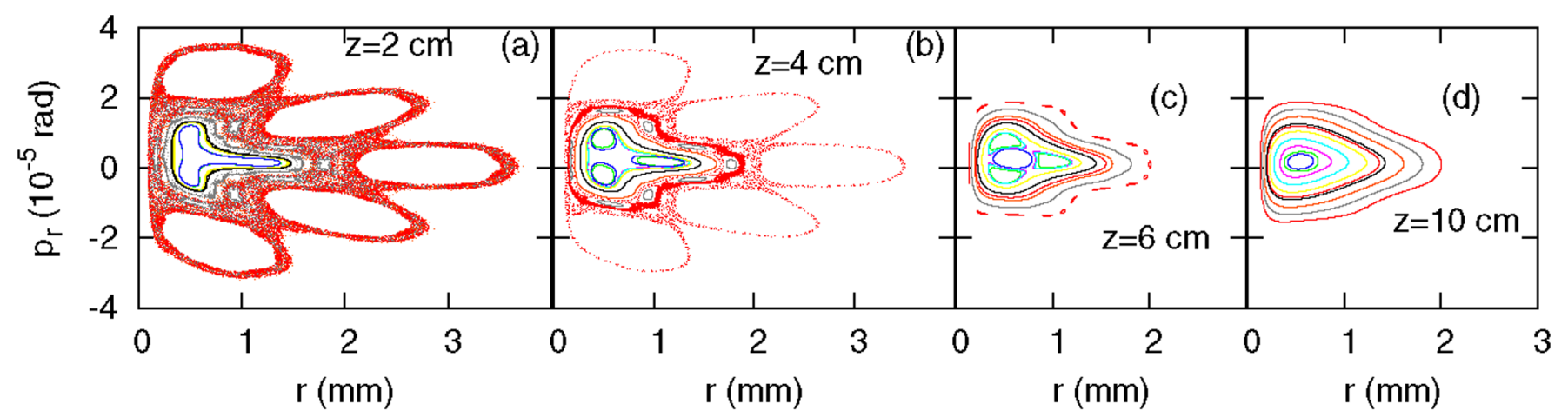

FIG. 6. (Color) Poincaré plot in $r-p_{r}$ for $z=2,4,6,10 \mathrm{~mm}(\xi=0.38)$. 
For the lowest tune shift $(\xi=0.06)$, the phase space plot consists of solid curves for every $z$ (the figure is not shown). This indicates that a surface in $r-p_{r}-z$ space , which is characterized by a function, $f\left(r, p_{r}, z\right)=$ const, exists; that is, the system is near integrable. The Poincaré plot in Fig. 5(a) seems to be the superposition of similar curves.

A three-dimensional plot gives direct information about whether the system is near integrable or stochastic. Using recent 3-dimensional graphic tools, the judgment of integrability is easy except for critical rare cases, because the viewpoint can be controlled dynamically. Trajectories in $r-p_{r}-z$ space of three particles with tune shifts of $\xi=$ 0.13 and 0.27 are plotted in Fig. 7. Red and blue points are trajectories with and without synchrotron motion $(z=0)$, respectively. Figure 7(a) shows an integrable example, i.e., a clear surface is seen. All particles at $\xi=0.06$ have the feature of integrability. While the trajectory without synchrotron motion at $z=0$ is a clear solid curve, that with synchrotron motion is also integrable. In this case, particles move on the surface determined by the initial conditions. Figure 7(b) shows a stochastic example. When the trajectory without synchrotron motion is stochastic, the stochastic nature is spread out to all $z$. Figure 7(c) shows another stochastic example. The trajectory without synchrotron motion is near integrable, but has island structures. With the variation of $z, r-p_{r}$ trajectory transferred from an island structure to a regular one. At the transition particles cross an unstable fixed point or small stochastic area, with the result that the whole $r-p_{r}-z$ trajectory becomes stochastic.

The evolution of $J_{r}$ reflects the trajectory in $J_{r}-\psi_{r}-z$ space and is directly related to the emittance growth. $J_{r}$ gives a flat surface for $U=0$, while $J_{r}=J_{r}\left(\psi_{r}, z\right)$ oscillates for a near integrable trajectory. The evolution of $J_{r}$ is expected to show a regular oscillation within a certain range for a near integrable trajectory.

Figure 8 shows the evolution of $J_{r}$ of 10 particles. Figures 8(a) and 8(b) depict the evolution with and without

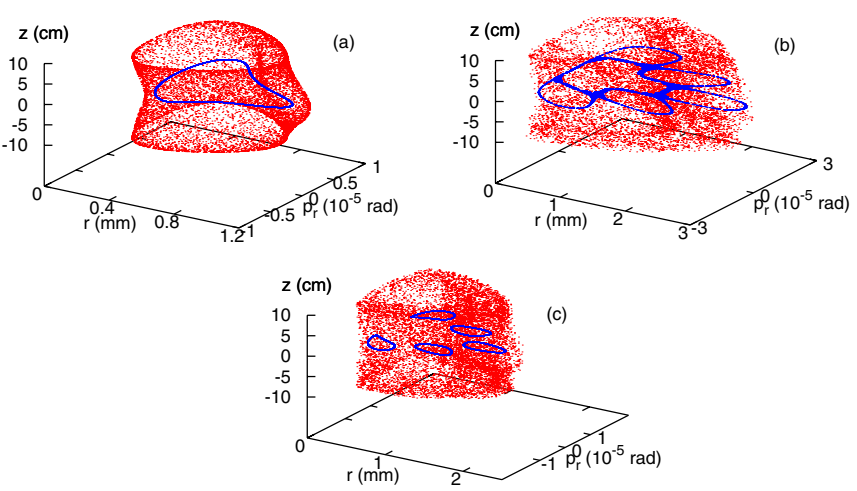

FIG. 7. (Color) Poincaré plot in $r-p_{r}-z$ space: (a) near integrable $(\xi=0.27)$, (b) stochastic $(\xi=0.27)$, (c) stochastic $(\xi=0.13)$.

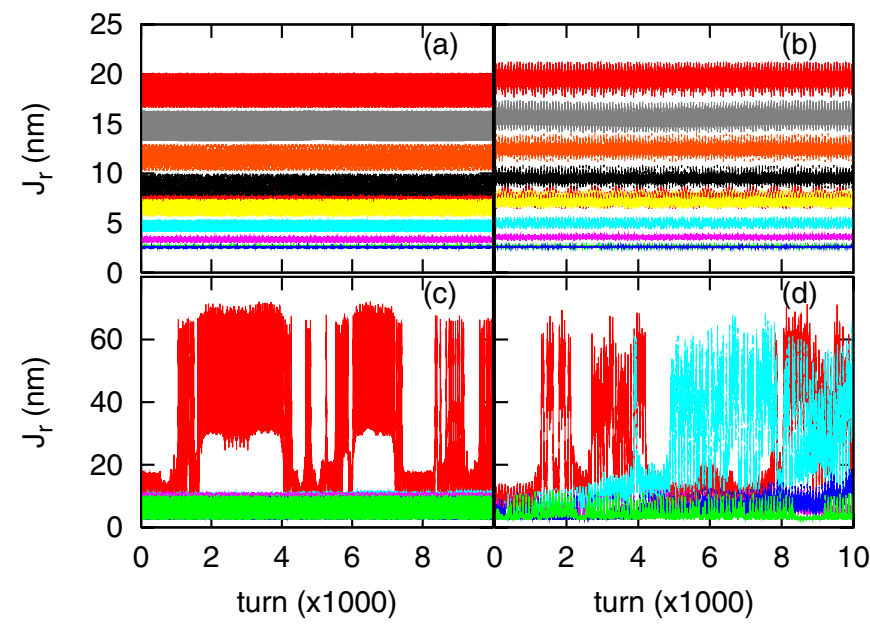

FIG. 8. (Color) Evolution of $J_{r}$ : (a) $\xi=0.06, \nu_{s}=0$, (b) $\xi=$ $0.06, \nu_{s}=0.006$, (c) $\xi=0.38, \nu_{s}=0$, (d) $\xi=0.38, \nu_{s}=$ 0.006 .

synchrotron motion, respectively, for $\xi=0.06$. A modulation of $J_{r}$ is seen due to the distortion of $J_{r}\left(\psi_{r}, z\right)$ in Fig. 8(b). Figures 8(c) and 8(d) depict the evolution with and without synchrotron motion, respectively, for $\xi=$ 0.38 . $J_{r}$ for some particles repeatedly increases and decreases with a roughly 1000-turn period for $\nu_{s}=0$ in Fig. 8(c). They sometimes travel to the island part shown in Fig. 3. For $\nu_{s}=0.006, J_{r}$ of some particles, marked by blue and light blue, increase only in Fig. 8(d). Some particles, which were nearly integrable without synchrotron motion, go into the stochastic layer due to the synchrotron oscillation, with the result that they contribute to emittance growth.

The chaotic transverse motion slightly influences the synchrotron motion. Figure 9 shows the evolution of $J_{z}$. The small effect even for $\xi=0.38$, which is only $0.1 \%$, is

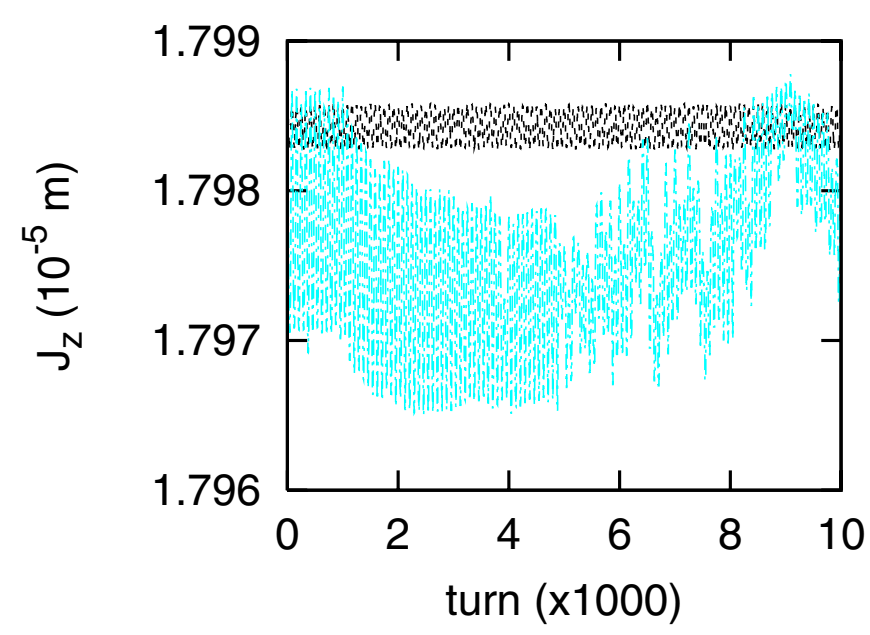

FIG. 9. (Color) Evolution of $J_{z}$ for the particle marked light blue in Fig. 8. Black and light blue lines correspond to $\xi=0.06$ and 0.38 , respectively. 
due to the difference of the transverse and longitudinal emittances, $\varepsilon_{x}=8 \times 10^{-9} \mathrm{~m}$ and $\varepsilon_{z}=6 \times 10^{-5} \mathrm{~m}$.

Since the transverse motion has little effect on the longitudinal motion, we have an approximate integral for synchrotron oscillation,

$$
2 J_{z}\left(z, p_{z}, s\right)=\beta_{z} p_{z}^{2}+z^{2} / \beta_{z}=\text { const, }
$$

where $\beta_{z}=\alpha / \mu_{z}$. The synchrotron oscillation is solved as

$$
z(s)=\sqrt{\beta_{z} J_{z}} \cos \mu_{z} s .
$$

The transverse force, which is given by $U(r, z)$, is modulated by $z$ as a function of $s$.

$U$ is a periodic function of $s$ with frequency $\mu_{z}$ :

$$
U(r, s)=\frac{N r_{e}}{\gamma} \sum_{k=0}^{\infty} \sum_{l=-\infty}^{\infty} U_{k l}\left(J_{r}\right) \cos \left(k \psi_{r}+l \psi_{z}\right)
$$

The resonance condition given in Eq. (23) is now revised as

$$
\bar{\mu}\left(J_{r, R}\right)+l \mu_{z}=2 \pi n / k
$$

The resonance width is

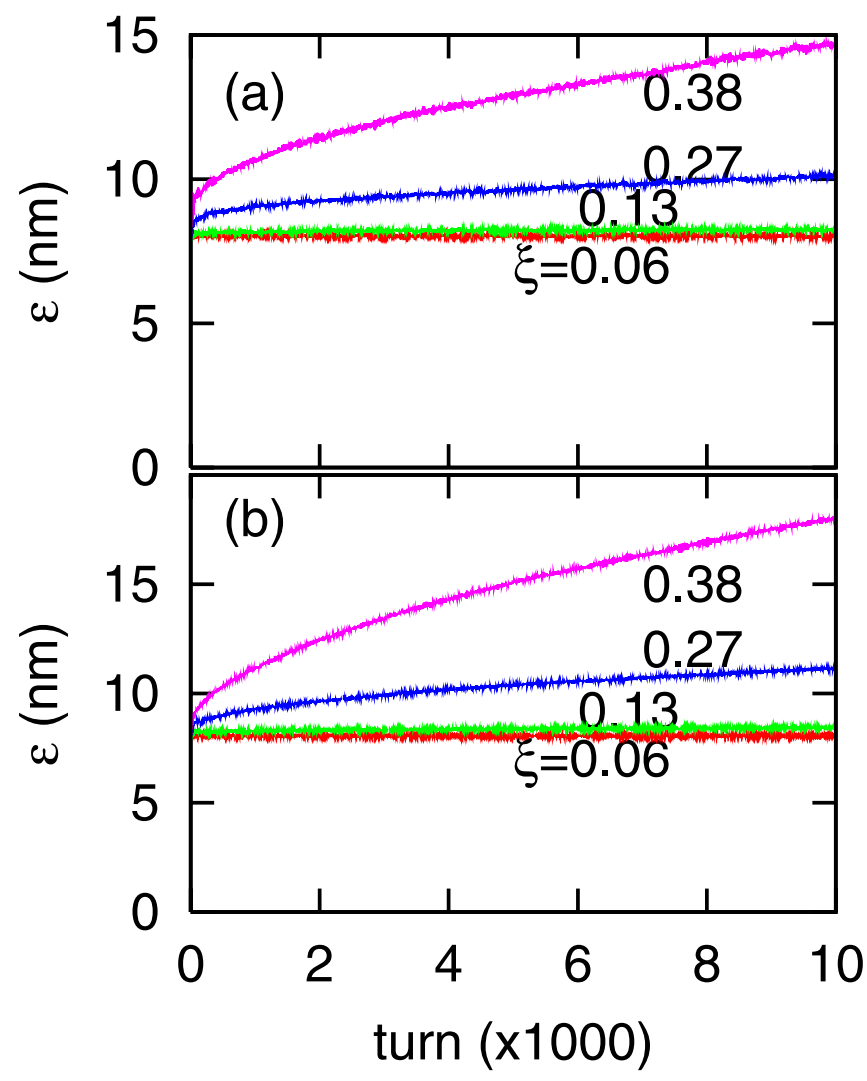

FIG. 10. (Color) Emittance growth for system with 3 degrees of freedom; transverse motion with equal tunes $\left(\nu_{x}=\nu_{y}=\right.$ 0.2901) and synchrotron motion. Synchrotron tunes for plots (a) and (b) are $\nu_{s}=0.006$ and 0.012 , respectively.

$$
\Delta J_{r}=k \Delta I_{r}=4 \sqrt{U_{k l}\left(\frac{\partial^{2} U_{0}}{\partial J_{r}^{2}}\right)^{-1}} .
$$

Now the resonance separation is determined by different values of $l$, i.e., an overlap between synchrotron sidebands induces a stochastic layer. The separation between sidebands is expressed by

$$
\left|J_{r, R}(k, l+1)-J_{r, R}(k, l)\right|=\frac{\mu_{z}}{k}\left(\frac{\partial^{2} U_{0}}{\partial J_{r}^{2}}\right)^{-1} .
$$

The overlap criterion is given as

$$
\frac{4 k}{\mu_{z}} \sqrt{U_{k l} \frac{\partial^{2} U_{0}}{\partial J_{r}^{2}}}>1 .
$$

A resonant overlap between sidebands can arise easily, because of the small $\mu_{z}$, with the result that the stochastic area extends and emittance growth occurs. This type of resonance overlap is called "separatrix crossing" [7,8].

Emittance growth is evaluated by multiparticle tracking for the system with synchrotron oscillation. Figure 10 shows the emittance growth for synchrotron tunes of $\nu_{s}=$ 0.006 and 0.012. Emittance increases monotonically for high tune shifts, $\nu_{\beta} \geq 0.27$. The emittance growth is clearly enhanced by the synchrotron motion as compared with Fig. 4. The growth is stronger for a larger synchrotron tune in comparing these two results.

\section{B. Difference of horizontal and vertical tunes}

The other way to increase the number of degrees of freedom is the introduction of a difference between the horizontal and vertical tunes. The Hamiltonian is expressed as follows:

$$
\begin{aligned}
H= & \mu_{x} J_{x}+\mu_{y} J_{y}+\delta_{P}(s) U(r, z) \\
= & \frac{\mu}{2}\left[\beta\left(p_{r}^{2}+\frac{p_{\theta}^{2}}{r^{2}}\right)+\frac{r^{2}}{\beta}\right] \\
& +\delta \mu \frac{r^{2}}{\beta} \cos 2 \theta+\delta_{P}(s) U(r, z),
\end{aligned}
$$

where $\delta \mu=\mu_{x}-\mu_{y}$. Since the Hamiltonian contains $\theta$ explicitly, $p_{\theta}$ is not invariant now. The variation of $p_{\theta}$ is given by

$$
p_{\theta}=p_{\theta}(0) \cos \delta \mu s .
$$

The motion can be characterized by five variables, $r, p_{r}, \theta, p_{\theta}, s$. We take the tune difference as small. The oscillation of $\theta-p_{\theta}\left(J_{\theta}\right)$ is slower than that of $r-p_{r}$. The centrifugal force, $p_{\theta}^{2} / r^{2}$, which is not constant now, influences the radial motion $r-p_{r}$. We may expect that the motion in $\theta$ is regarded as a slow external motion similar to the synchrotron motion. However, $r-p_{r}$ motion influences the variation of $p_{\theta}$, because the two emittances $\left(\varepsilon_{x}\right.$ and $\varepsilon_{y}$ ) are the same for the transverse motion. 

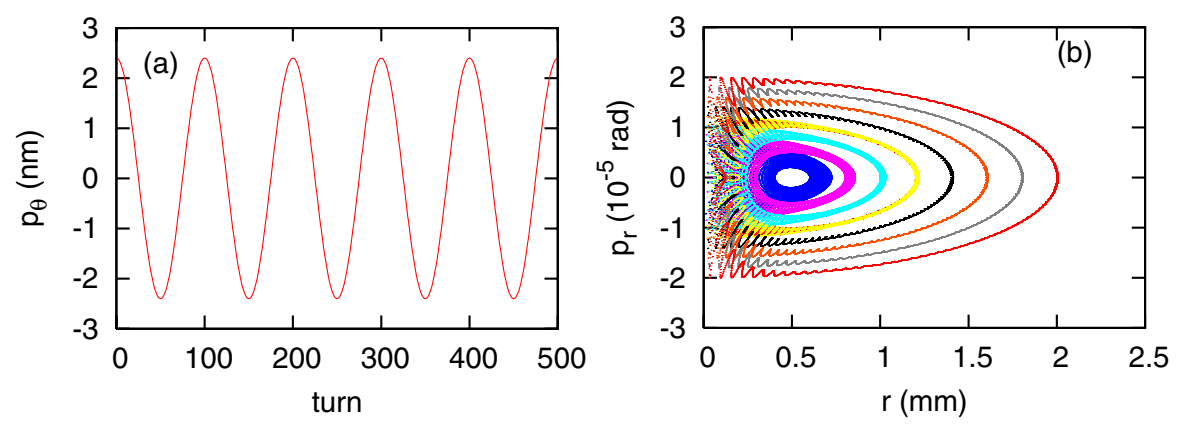

FIG. 11. (Color) Poincaré plot in $r-p_{r}$ space and evolution of $p_{\theta}$ for different transverse tunes, $n u_{x}=0.285$ and $\nu_{y}=0.295$ at $U=0$.

$J_{r}$ and $J_{\theta}$ are defined as invariants at $\delta \mu=0$ and $U=0$. $J_{\theta}$ and $J_{r}$ wave for $\psi_{r}$ and $\psi_{\theta}$ due to $\delta \mu$ even for $U=0$. When the system is integrable, two integrals exist as $f_{1,2}\left(r, p_{r}, \theta, p_{\theta}\right)=\mathrm{const}$ or $g_{1,2}\left(J_{r}, \psi_{r}, J_{\theta}, \psi_{\theta}\right)=$ const. Figure 11 shows the evolution of $p_{\theta}$ and the Poincaré plot in $r-p_{r}$ space for $U=0$, where the transverse tunes are $\nu_{x}=0.285$ and $\nu_{y}=0.295 . p_{\theta}$ oscillates according to the tune difference, 0.01. The $r-p_{r}$ plot in Fig. 11(b) does not show a solid curve due to $p_{\theta}$ variation, though the system is integrable. In $r-p_{r}-\theta$ space, the particle trajectory remains on a surface. The distributed structure is due to the projection of $\left(r, p_{r}\right)$ along $\theta$.

Figure 12 shows the Poincaré plot for tune shift values, $\xi=0.06$ and $\xi=0.38$. If the system is near integrable, a torus is drawn in $r-p_{r}-\theta$ space. Since the $r-p_{r}$ position is projected in $\theta$ in this figure, it is difficult to distinguish whether the system is stochastic or near integrable.

In $J_{r}-\psi_{r}-\theta$ or $J_{\theta}-\psi_{r}-\theta$ space, $J_{r}\left(\psi_{r}, \theta\right)$ or $J_{\theta}\left(\psi_{r}, \theta\right)$ remains on a surface for an integrable system. The evolution of $J_{r}$ and $J_{\theta}$ gives information on whether the system is near integrable or stochastic. $J$ 's oscillate within a range for near integrable systems, while they behave nonperiodic and diffusive for stochastic systems.

Figure 13 shows the evolution of (a) $J_{\theta}=p_{\theta}$ and (b) $J_{r}$ for $\xi=0.27$. $\theta$ of some particles oscillate with a regular frequency, while others are diffusive and their amplitudes increase. This behavior is in contrast with that of $J_{z}$ shown in Fig. 9. $J_{r}$ have similar behavior to $p_{\theta}$. Each particle trajectory is plotted in both plots with the same color.

The horizontal and vertical tunes are calculated as the betatron phase advance of each particle turn by turn, i.e., $\Phi_{x}=\tan ^{-1}\left(\beta_{x} p_{x} / x\right)$ and $\Phi_{y}=\tan ^{-1}\left(\beta_{y} p_{x} / y\right)$. Figure 14 shows tune variation and the corresponding $r-p_{r}$ plot. Particle motions are classified as near integrable or stochastic. Figures 14(a) and 14(c) depict tune variation and phase space trajectory in $r-p_{r}$ plane for near integrable trajectories, while Figs. 14(b) and 14(d) depict them for stochastic ones. Each trajectory is plotted with the same color as those of Fig. 13.

As mentioned, a three-dimensional plot gives direct information on whether the system is near integrable or stochastic. Figure 15 shows the trajectory in $r-p_{r}-p_{\theta}$ space. Figure 15(a) is a near integrable example which corresponds to the blue trajectory in Figs. 14(a) and 14(c), while Fig. 15(b) is a stochastic example which corresponds to the light blue trajectory in Figs. 14(b) and 14(d).

The mechanism of emittance growth is more complex than that due to synchrotron motion in Sec. IVA. The Poincaré plot of $r-p_{r}$ phase space is changed by the variation of $p_{\theta}$ as is shown for synchrotron motion in
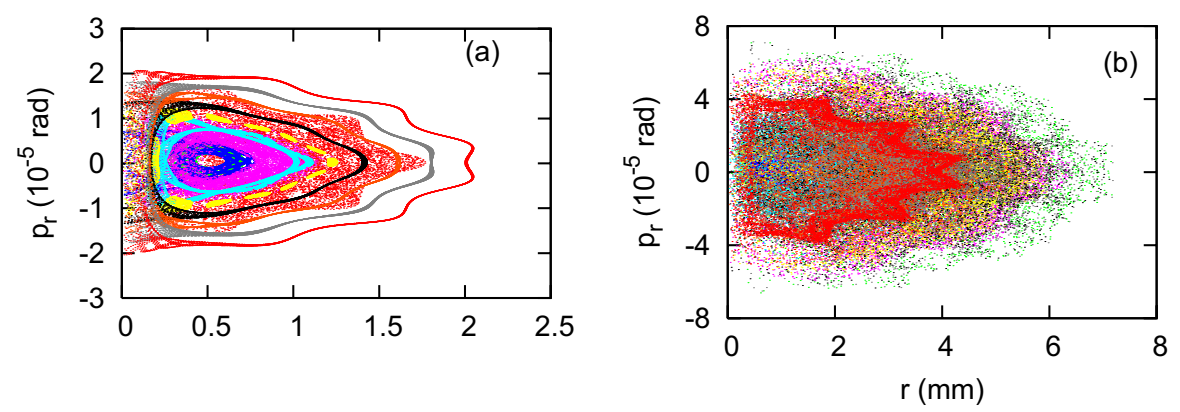

FIG. 12. (Color) Poincaré plot in $r-p_{r}$ space for different horizontal tunes, $\nu_{x}=0.285$ and $\nu_{y}=0.295$. Tune shifts are (a) $\xi=0.06$ and (b) $\xi=0.38$. 


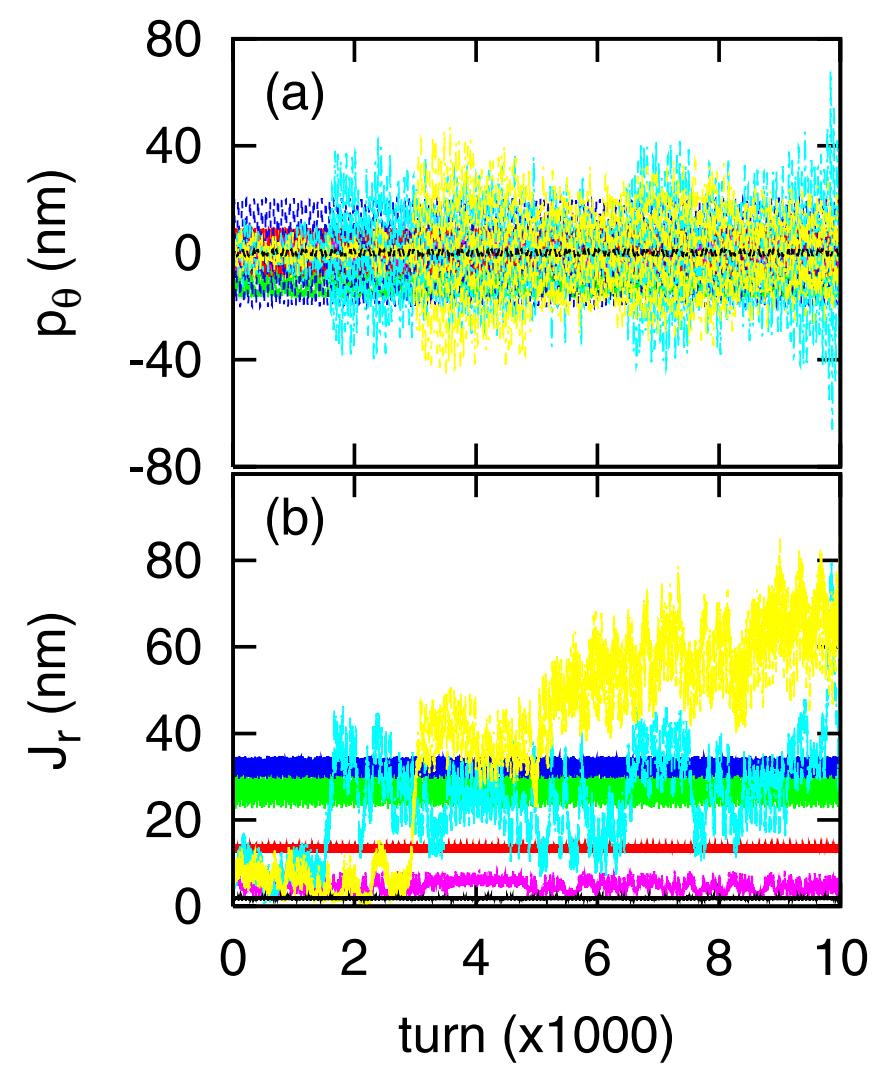

FIG. 13. (Color) Evolution of $p_{\theta}$ and $p_{r}$ of ten sampled particles for $\nu_{x}=0.285, \nu_{y}=0.295$, and $\xi=0.27$. Each trajectory is plotted in both plots with the same color.
Fig. 6. Even if the $p_{\theta}$ motion is regarded as an external regular oscillation, stochasticity of $r-p_{r}$ motion is enhanced by the separatrix crossing.

The stochastic nature in $r-p_{r}$ space gives a diffusion in $p_{\theta}$ motion via the coupling term, $p_{\theta}^{2} / r^{2}$. This mechanism is called stochastic pumping [9]. Figure 13(a) show the diffusion of $p_{\theta}$ due to stochastic pumping. The induced stochasticity in $p_{\theta}$ gives back diffusion in $r-p_{r}$ motion again.

Coupling resonance is another important mechanism for causing emittance growth. The Hamiltonian is expressed as

$$
\begin{aligned}
H= & \mu_{x} J_{x}+\mu_{y} J_{y}+\delta_{P}(s) \sum_{k_{x}, k_{y}=0}^{\infty} U_{k_{x}, k_{y}}\left(J_{x}, J_{y}\right) \\
& \times \cos \left(k_{x} \psi_{x}+k_{y} \psi_{y}\right) .
\end{aligned}
$$

The tune shift is given by

$$
\mu_{x}\left(J_{x}, J_{y}\right)=\mu_{x}+\frac{\partial U_{00}}{\partial J_{x}} \quad \mu_{y}\left(J_{x}, J_{y}\right)=\mu_{y}+\frac{\partial U_{00}}{\partial J_{y}} .
$$

The resonance condition is

$$
\mu_{x}\left(J_{x}, J_{y}\right) k_{x}+\mu_{y}\left(J_{x}, J_{y}\right) k_{y}=2 \pi n .
$$

This condition gives a line in $\left(J_{x}, J_{y}\right)$ space. New variables are introduced to characterize the motion near the resonance as
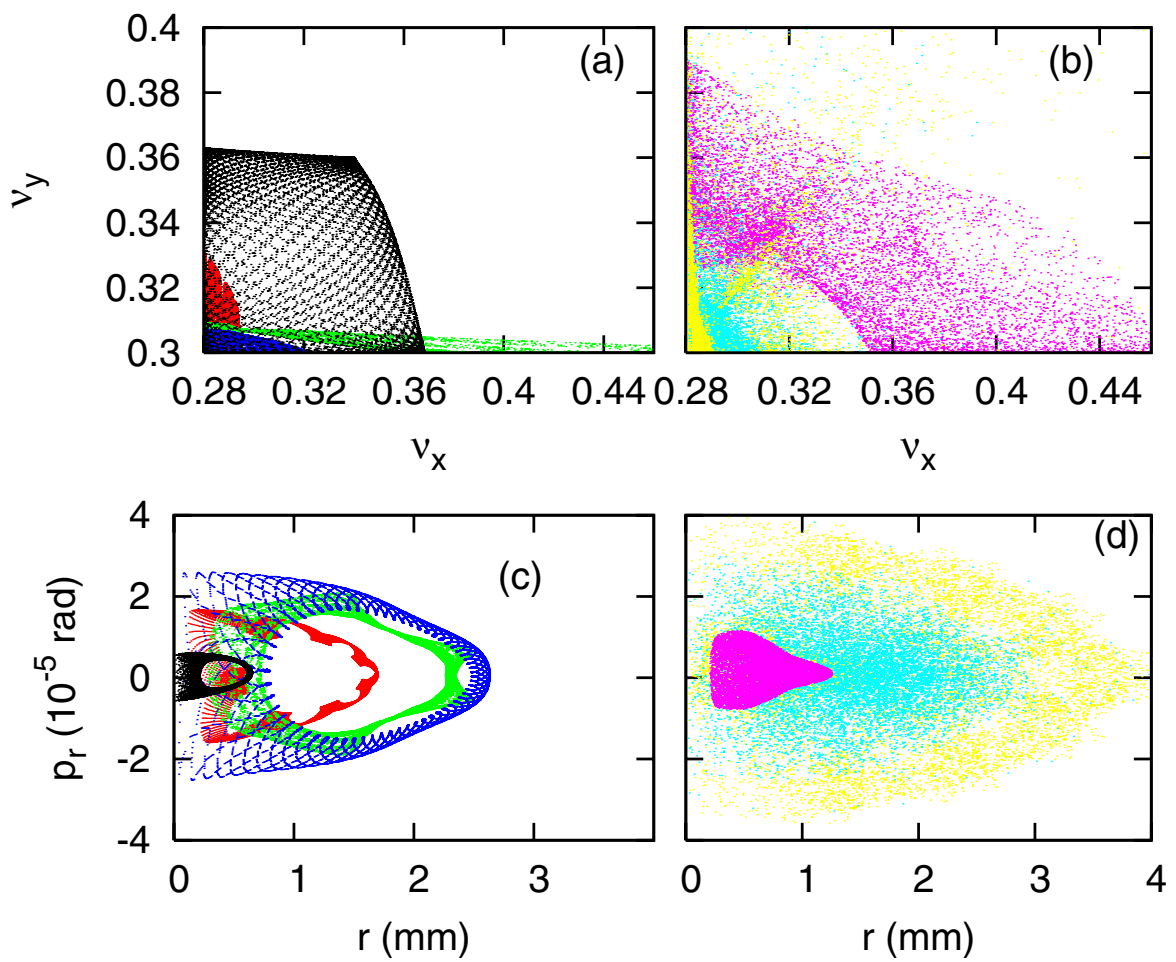

FIG. 14. (Color) Variation of horizontal and vertical tunes, and $r-p_{r}$ phase space motion. Plots (a) and (b) depict tune variation for near integrable and stochastic particles, respectively. Plots (c) and (d) are the corresponding $r-p_{r}$ plots to (a) and (b), respectively. 

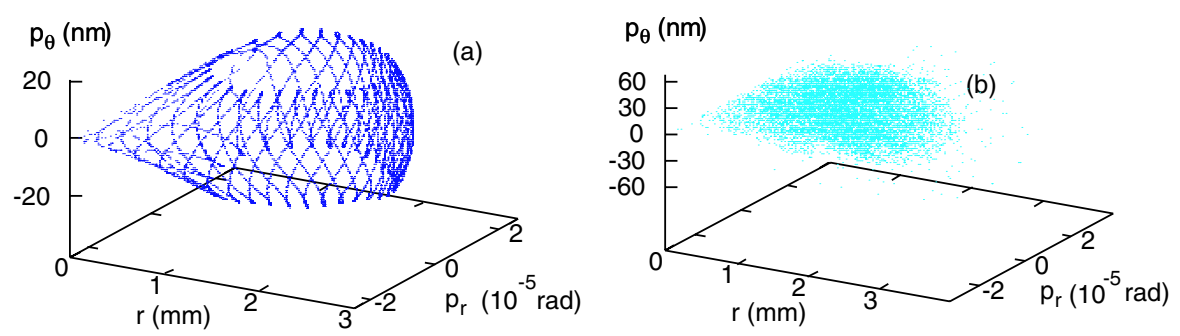

FIG. 15. (Color) Poincaré plot in $r-p_{r}-p_{\theta}$ space. Plots (a) and (b) are near integrable $(\xi=0.27)$ and stochastic $(\xi=0.27)$ trajectories, respectively. The trajectories of blue and light blue correspond to those of Fig. 14 plotted with the same colors.

$$
\begin{array}{ll}
k_{x} I_{1}+l_{x} I_{2}=J_{x}-J_{x, R} & \theta_{1}=k_{x} \psi_{x}+k_{y} \psi_{y}, \\
k_{y} I_{1}+l_{y} I_{2}=J_{y}-J_{y, R} & \theta_{2}=l_{x} \psi_{x}+l_{y} \psi_{y},
\end{array}
$$

where $l_{x}$ and $l_{y}$ are not specified. The Hamiltonian near the resonance is expressed as

$$
H=\frac{\Lambda_{11}}{2} I_{1}^{2}+\delta_{P}(s) U_{k_{x}, k_{y}} \cos \left(\theta_{1}\right)
$$

where

$$
\Lambda_{11}=\frac{\partial^{2} U_{00}}{\partial J_{x}^{2}} k_{x}^{2}+2 \frac{\partial^{2} U_{00}}{\partial J_{x} J_{y}} k_{x} k_{y}+\frac{\partial^{2} U_{00}}{\partial J_{y}^{2}} k_{y}^{2}
$$

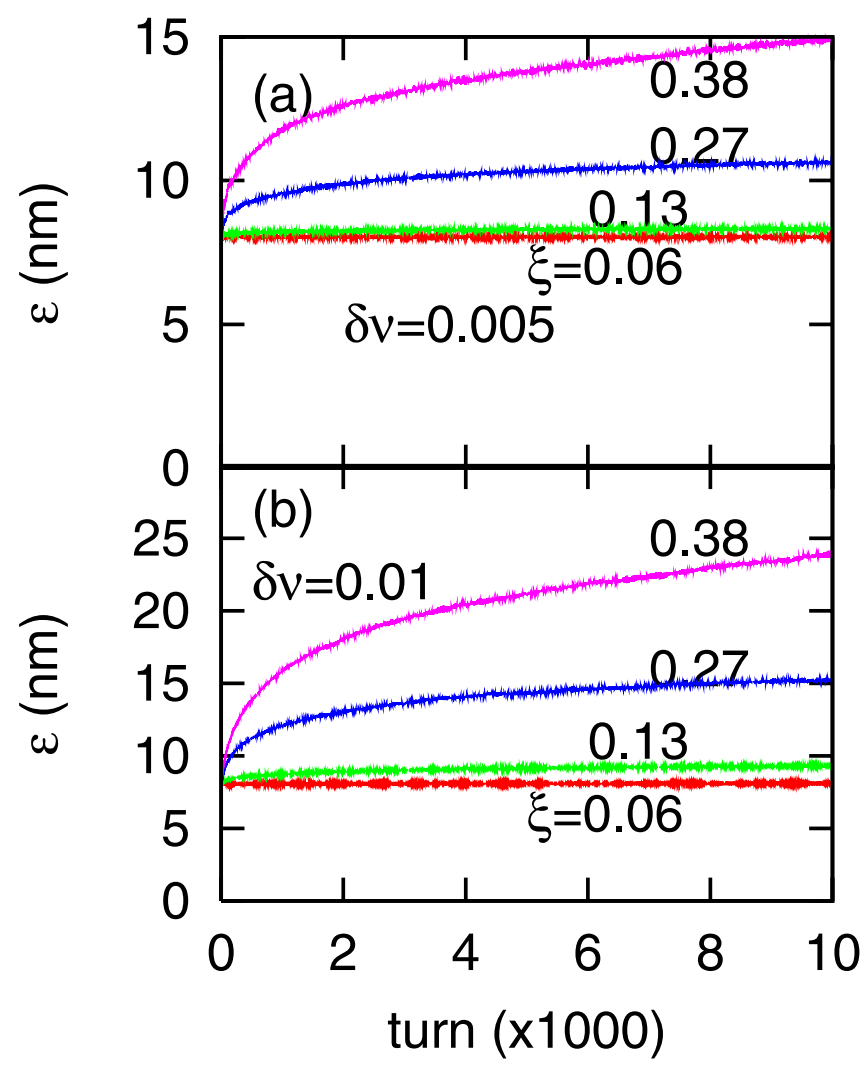

FIG. 16. (Color) Emittance growth for the system with 3 degrees of freedom; (a) $\left(\nu_{x}, \nu_{y}\right)=(0.2876,0.2926)$ and (b) $(0.2851$, 0.2951). Particles do not experience synchrotron motion.
The derivatives of $U_{0}$ are evaluated at $J_{x}=J_{x, R}$ and $J_{y}=$ $J_{y, R}$. The Hamiltonian shows a pendulum motion around the resonance. There are several reasons why the coupling resonance is more serious than the single resonance. The coupling resonance condition $\left(k_{x} \mu+k_{y} \mu_{y}=2 \pi n\right)$ is satisfied on more occasions than the single resonance $(k \mu=$ $2 \pi n)$. The Hamiltonian, Eq. (45) or Eq. (40), does not describe the motion for $I_{2}$. Motion along $I_{2}$, which gives emittance growth, is called resonance streaming.

Emittance growth is again evaluated by multiparticle tracking. Figure 16 shows the emittance growth for transverse tunes of $\left(\nu_{x}, \nu_{y}\right)=(0.2876,0.2926)$ and $(0.2851$, $0.2951)$. Emittance increases monotonically again. The emittance growth is stronger for larger tune differences as compared with the results for small tune differences. The growth rate is similar to that with round symmetry and synchrotron motion in Fig. 10.

\section{SYSTEM WITH FOUR DEGREES OF FREEDOM}

We now go to a more complex system, in which the transverse tunes are different and the synchrotron tune is finite. The synchrotron oscillation can, again, be considered as an external modulation for the transverse motion.

The Poincare section surface is represented in the 6dimensional space of $\left(x, p_{x}, y, p_{y}, z, p_{z}\right)$ or $\left(r, p_{r}, \theta, p_{\theta}\right.$, $z, p_{z}$ ). If we have three integrals, the system is integrable. The solution describes a surface in four-dimensional space, like $J_{r}\left(\psi_{r}, \psi_{\theta}, z\right)$. Needless to say, it is difficult to image and analyze whether the surface exists or not (near integrable or stochastic). Therefore we do not visualize the integrability, but show only results for multiparticle tracking here.

Figure 17 shows emittance growth for various tune shift values. Even for the lowest tune shift, $\xi=0.06$, considerable emittance growth is seen in Fig. 17(b).

The system with 4 degrees of freedom can be regarded as one with 3 degrees of freedom in $x$ and $y$, with synchrotron modulation as an external motion. For 3 degrees of freedom in $x$ and $y$ in Sec. IV B, pumping and resonance streaming was the source of diffusion. The diffusion is further enhanced by the synchrotron modulation, that is, the resonance width is spread and the stochasticity is enlarged. This is called modulation diffusion. 


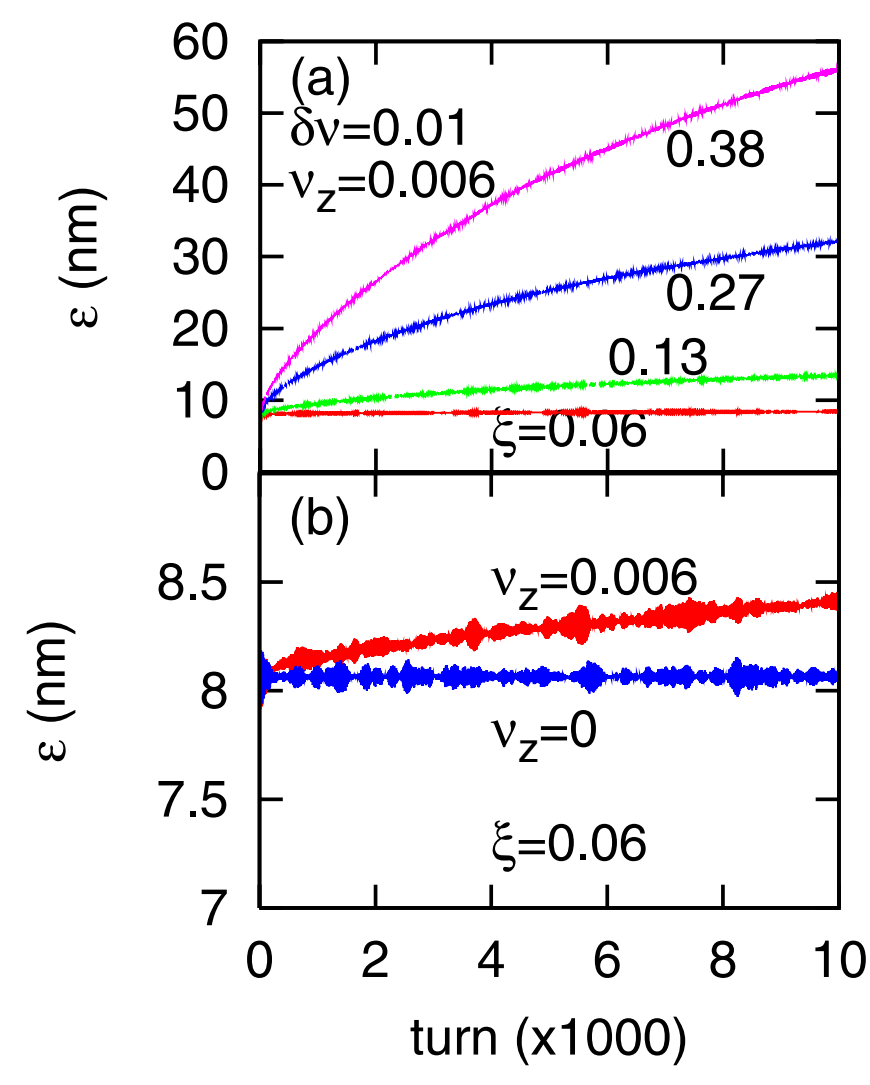

FIG. 17. (Color) Emittance growth for system with 4 degrees of freedom, where $\left(\nu_{x}, \nu_{y}, \nu_{z}\right)=(0.2851,0.2951,0.006)$. The growths for $\xi=0.06-0.38$ are depicted in plot (a), and that for $\xi=0.06$ is depicted in plot (b) with a fine scale.

We take into account an arbitrary tune set in the transverse plane. The emittance growth strongly depends on the tune, especially the transverse tune. Emittance growth rate is calculated in tune space $\left(0.05<\nu_{x}, \nu_{y}<0.45\right)$ with steps of 0.01 . Figure 18 shows the diffusion rate in the transverse tune space. Figures 18(a) and 18(b) depict the rate with and without synchrotron oscillation, respectively. Some resonance lines are seen in the figure. The resonance lines are prominent for the system with synchrotron motion. At the crossing point of resonances the diffusion is strongly enhanced.

\section{REALISTIC MODEL}

So far, we have discussed the case in which the beam and electron cloud interact at a certain longitudinal position in a ring. Actually the electron cloud is distributed along the whole ring. Therefore the nonlinear interaction should be evaluated along the ring [10]. Variations of betatron phase, Twiss, and dispersion parameters have to be taken into account to integrate the interaction along the ring.

Beam particles are tracked along the lattice element by element while evaluating the beam-electron cloud interactions. Since the tracking is very time consuming, we use a simplified model. In many rings, a cell structure with a

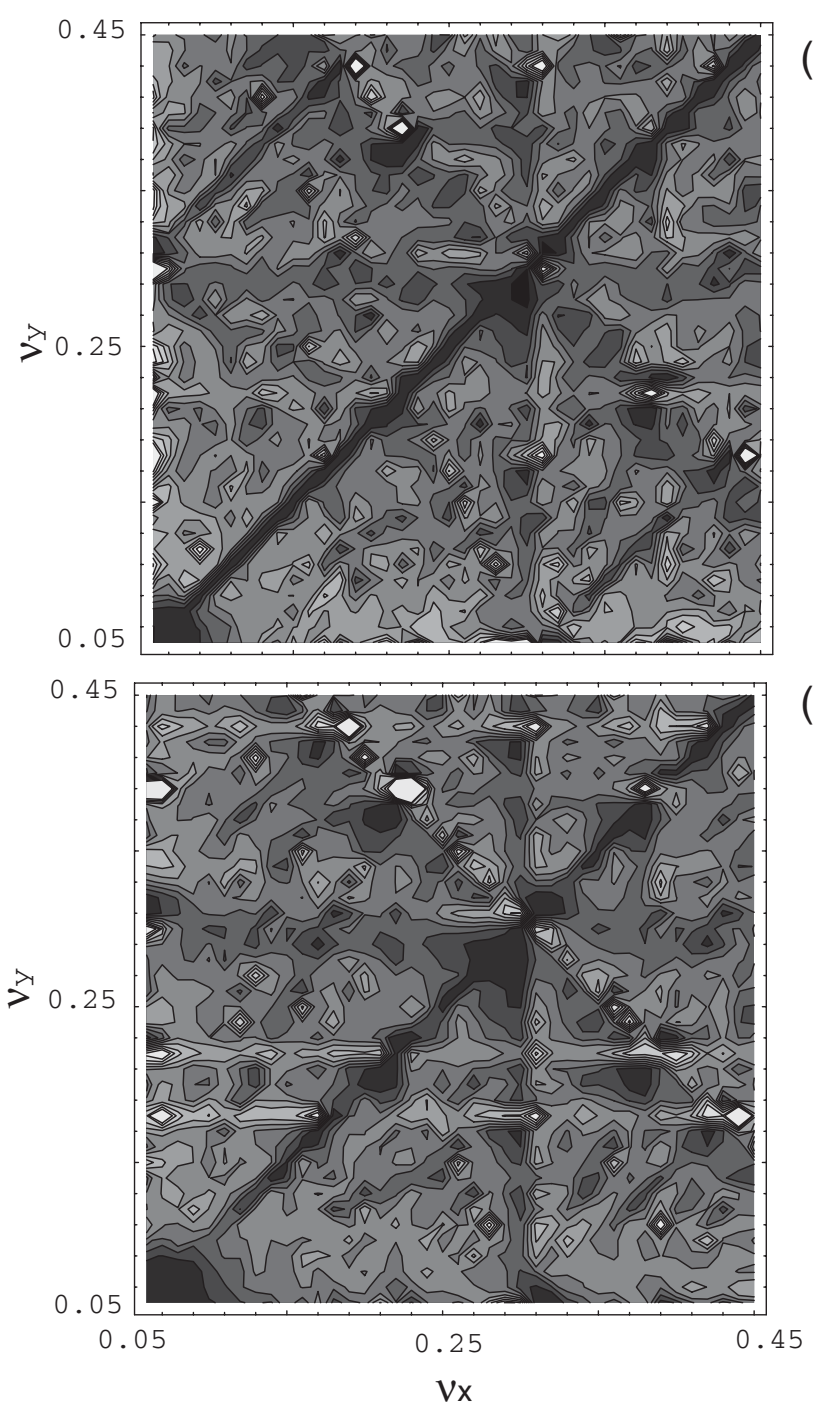

(a)

(b)

FIG. 18. Diffusion rate in transverse tune space. Plots (a) and (b) are given for $\nu_{s}=0$ and $\nu_{s}=0.006$, respectively. The contour lines are drawn every $D=1 \times 10^{-5}$, and the area labeled white is the highest rate, $D=1 \times 10^{-4}$ in plot (a). The lines are drawn every $D=3 \times 10^{-5}$, and the area labeled white is the highest value, $D=3 \times 10^{-4}$ in plot (b).

certain betatron phase advance is repeated. The contribution of the nonlinearity is evaluated as a simple multiplication for the repetition of a combination of cells with $2 \pi$ phase advance. We approximate the ring with a series of cells with a betatron phase advance of $2 \pi$. A one-turn map is expressed for the whole ring which consists of $N$ structures repetition as

$$
\begin{aligned}
M & =e^{-: F_{\mathrm{fr}}}:\left(\prod_{\text {cell }} e^{-: F_{i+1, i}:} e^{-: U\left(s_{i}\right):}\right)^{N} \\
& =e^{-: F_{\mathrm{fr}}} \prod_{\text {cell }} e^{-: F_{i+1, i}:} e^{-N: U\left(s_{i}\right)},
\end{aligned}
$$

where $e^{-: F_{\mathrm{fr}}:}$ is the linear map to adjust the fractional tune 


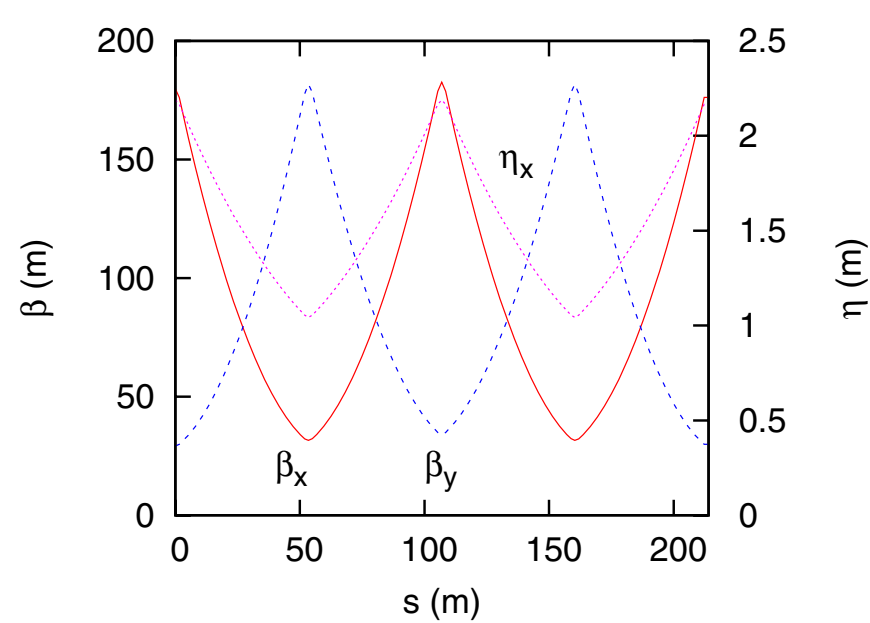

FIG. 19. (Color) Optics functions of the arc section (two cells) of LHC. The betatron phase advance is 180 degrees in both horizontal and vertical.

of the ring. The product is the integral of the beam-electron cloud interaction along the $2 \pi$ structure, where $e^{-F_{i+1, i}}$ is the linear map from $s_{i}$ to $s_{i+1}$. Since the beam-electron cloud potential $\phi$ is symmetric for $x \rightarrow-x$ and $y \rightarrow-y$, it is enough to consider a structure with $\pi$ phase advance. Using this approximation, computation time can be drastically reduced.

Figure 19 shows Twiss parameters for a structure with two cells of an LHC arc. The phase advance is $\pi$ for both $x$

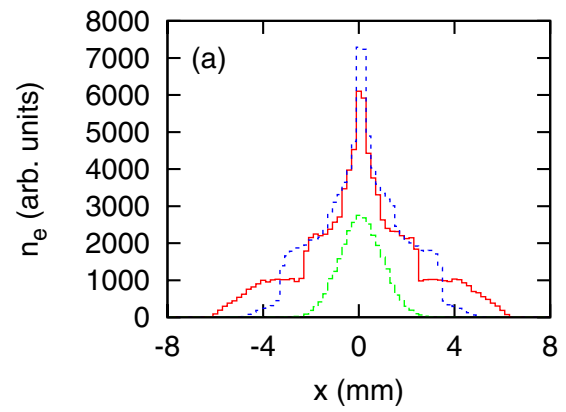

and $y$. The whole ring is assumed to consist of $125 \times 2$ cells and an additional section to match the operating tune. Super periodicity of the lattice is not considered.

For the beam-electron cloud interaction, we use a more realistic model with a simulation code (PEHTS) based on the so-called strong-strong model. The beam (bunch) and electron cloud are represented by 500 and $100 \mathrm{k}$ macroparticles, respectively, and the interaction is evaluated by the particle-in-cell method [11]. In the simulation, electrons are initialized with a flat distribution in the transverse region $16 \times 16 \mathrm{~mm}^{2}$, while macroparticles in a bunch are initialized with a Gaussian distribution determined by the emittance and the beta and dispersion functions. The electron cloud is initialized at every interaction, while the macroparticles in the bunch are transferred with the nonlinear interaction $(\phi)$ and a linear matrix.

Figure 20(a) shows the transverse profile of the beam and electron cloud during the interaction. The peak electron cloud density, which is achieved slightly after the interaction with the bunch center $\left(z_{\text {peak }} \approx-0.2 \sigma_{z}\right)$, is 200 times higher than the initial value in the figure. Figure 20(b) shows emittance growth for interaction with electron cloud at a single location. The emittance growth ( $\xi=0.16)$ is compared with that of the model $(\xi=0.13)$ discussed in Sec. V. These results roughly agree with each other. The growth rate is $D=1.3 \times 10^{-4}$ for the interaction $(\xi=0.16)$ at a single location.

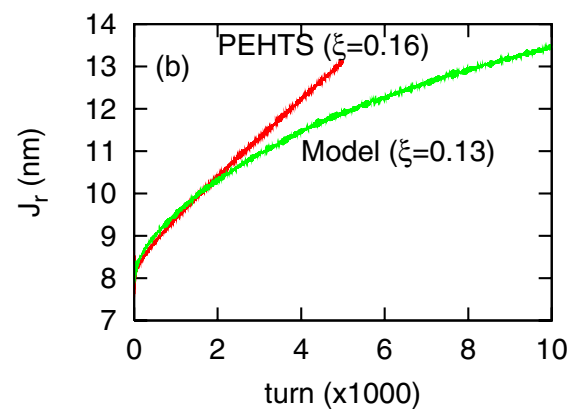

FIG. 20. (Color) Transverse electron distribution during the beam interaction [plot (a)] and emittance growth for single interaction point [plot (b)]. The profile of the beam (green) and electron cloud (red and blue) are plotted in plot (a). Emittance growth given by the PIC simulation and simple model in Sec. V are plotted with red and green lines in plot (b).
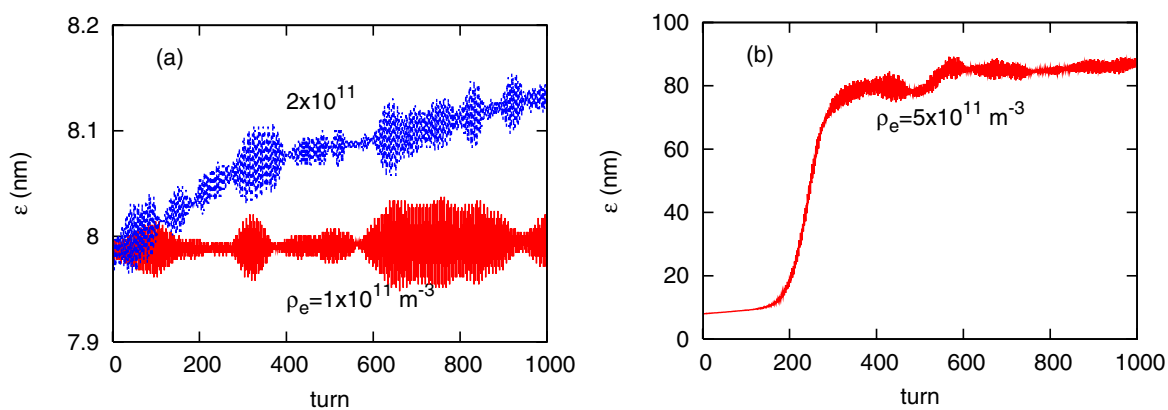

FIG. 21. (Color) Emittance growth for electron distribution along the lattice given in Fig. 19. A strong-strong simulation based on the particle-in-cell method is used. Plots (a) and (b) are $\rho_{e}=1,2 \times 10^{11}$ and $5 \times 10^{11} \mathrm{~m}^{-3}$, respectively. 
The same simulation is performed for the lattice model of Eq. (47) and Fig. 19. Figure 21 shows the emittance growth for the initial electron cloud density of $\rho_{e}=$ $1,2,5 \times 10^{11} \mathrm{~m}^{-3}$. The peak density, which is 200 times higher, corresponds to the tune shift of 0.08 for $\rho_{e}=1 \times$ $10^{11}$, i.e., $\xi=0.08,0.16,0.4$, respectively. For $\rho_{e}=5 \times$ $10^{11} \mathrm{~m}^{-3}$, a strong emittance growth is caused by onset of a coherent strong head-tail instability. Below the instability threshold, $\rho_{e}=2 \times 10^{11} \mathrm{~m}^{-3}$, an emittance growth is observed in Fig. 21(b). The growth rate is $D=$ $1.9 \times 10^{-5}$ for $\rho_{e}=2 \times 10^{11} \mathrm{~m}^{-3} \quad(\xi=0.16)$. The growth rate is reduced to $1 / 7$ of that in the case of a single location.

\section{CONCLUSION}

We have studied incoherent emittance growth due to electron clouds. In the first place, a simple model, which was based on the interaction with a fixed round charged distribution at a location of a circular accelerator, was used. The number of degrees of freedom of the interacting system was controlled by the choice of parameters, the synchrotron and transverse tunes. We started by studying a system with 2 degrees of freedom and extended to 4 degrees of freedom. The emittance growth was strongly enhanced by increasing the number of degrees of freedom. Integrability of the Hamiltonian was also profoundly related to the emittance growth. The emittance growth is caused by various diffusion mechanisms: the separatrix crossing, stochastic pumping, resonance streaming, and modulation diffusion. We discussed them qualitatively in this paper. A more quantitative discussion with analytic approaches will be done elsewhere.

More realistic simulations using a strong-strong model based on the particle-in-cell method were carried out. The simulation for an electron cloud at a single location gave agreement with the simple model. The other simulation for an electron cloud distributed along the ring gave slower emittance growth than that for a single location; i.e., the growth rate was reduced to $1 / 7$ in this parameter. The turn number is insufficient for the LHC operation. A longer term simulation should be done.

\section{ACKNOWLEDGMENTS}

The author thanks fruitful discussions with E. Benedetto, G. Franchetti, S. Kamada, S. Heifets, E. Perevedentsev, F. Zimmermann, and M. Zobov. They also thank J. Flanagan for reading the manuscript. This work is supported by the Large Scale Simulation Program No. 06-11 (FY2006) of KEK.

[1] B. V. Chirikov, Phys. Rep. 52, 263 (1979).

[2] J.L. Tennyson, Beam-Beam Interaction, in AIP Conference Proceedings No. 87 (AIP, New York, 1982), pp. 345-394.

[3] Nonlinear Dynamics Aspects of Particle Accelerators, Sardinia, 1985, edited by J. M. Jowett, M. Month, and S. Turner, Lecture Notes in Physics (Springer-Verlag, New York, 1985).

[4] E. Benedetto, D. Schulte, F. Zimmermann, and G. Rumolo, Phys. Rev. ST Accel. Beams 8, 124402 (2005).

[5] K. Ohmi, KEK Accelerator Physics Seminar, KEK, 2005.

[6] E. Benedetto, G. Franchetti, and F. Zimmermann, Phys. Rev. Lett. 97, 034801 (2006).

[7] J. R. Cary, D. F. Escande, and J. L. Tennyson, Phys. Rev. A 34, 4256 (1986).

[8] A. J. Lichtenberg and M. A. Lieberman, Regular and Chaotic Dynamics (Springer-Verlag, New York, 1992).

[9] K. Oide, KEK 96-149, 1996.

[10] K. Ohmi, 2005 ALCP \& ILC Workshop, Snowmass, 2005, ILCAW0424.

[11] K. Ohmi, Particle Accelerator Conference, 2001, pp. 1895-1897. 